\title{
Interest Point Detection - A Computer Vision Approach
}

\author{
Diptam Dutta \\ Computer Science \& \\ Engineering \\ Heritage Institute of \\ Technology
}

\author{
Priyanka Mukherjee \\ Computer Science \& Engineering \\ Institute of Engineering \& \\ Management
}

\author{
Sandeep Kumar Jha \\ Computer Science \& \\ Engineering \\ Heritage Institute of \\ Technology
}

\begin{abstract}
This paper describes a complementary mechanism that attempts to represent the Interest points (key points)[7][9][10] by a few of the intrinsic parameters in a rotation, scale and translation invariant manner. The parameter for this mechanism of finding interest point is that, the feature points or interest points[7][9][10] correspondences when the shapes of interest are each defined by a single, closed contour and the binary shape we obtained through segmentation represents some realworld object, which was sampled and binarized, and it is that object's identification that we want to estimate. That means by joining those key points, an image can be extracted. Corner is so special since it is the intersection of two edges; it represents a point in which the directions of these two edges change. Hence, the gradient of the image (in both directions) have a high variation, which can be used to detect it.
\end{abstract}

\section{Keywords}

Computer Vision, Object recognition, Image matching, Contour Tracing, Interest Point detection.

\section{INTRODUCTION}

Computer vision[1][2] is using a computer to capture and analyze images, also called "machine vision" (MV), there are numerous applications of computer vision, including robotic systems that sense their environment, people detection in surveillance systems, object inspection on an assembly line, image database organization and medical scans. In computer vision [1][2], object recognition is one of the vital task of finding a given object in an image or video sequence. Humans recognize a multitude of objects in images with little effort, despite the fact that the image of the objects may vary somewhat in different viewpoints, in many different sizes, scale or even when they are translated or rotated. Objects can even be recognized when they are partially obstructed from view. This task is still a challenge for computer vision systems in general. Henceforth, Image matching or comparing images in order to obtain a measure of their similarity, is a fundamental aspect of object and scene recognition. Image matching [8] can be defined as "the process of bringing two images geometrically into agreement so that corresponding pixels in the two images correspond to the same physical region of the scene being imaged". The goal is to detect objects in images using a single model and identify correspondences between the image and the model. Contour is used as token for image-model shape matching. In this paper a technique is proposed to find the points of interest viz. key points[7][9][10], which are mandatory for the representation of the contour. The contour information represents an extremely important and sensitive information for the human-visual system, being thus also of great importance in the image coding context. Finally the performance of the proposed algorithm is assessed by taking different images as input.

\section{LITERATURE REVIEW}

\subsection{Contour tracing}

Contour tracing[3][4] is a technique that is applied to digital images in order to extract their boundary, also known as border following or boundary following. The boundary of a given pattern $\mathrm{P}$ is the set of border pixels of $\mathrm{P}$. A digital image is a group of pixels on a square tessellation each having a certain value. A tessellation is created when a shape is repeated over and over again covering a plane without any gaps or overlaps. There are some considerations which are as follows: 1, in which case we'll consider it a "black" pixel and it will be part of the pattern, or 0 , in which case we'll consider it a "white" pixel and it will be part of the background. The need is an ordered sequence of the boundary pixels from which the general shape of the pattern can be extracted. The contour of the image will be more than one pixel thick, So thinning is performed. And If an image is seen in the computer i.e. in raster scale, the straight line will not appear as just like exactly the straight line like real plane, There will be Stair case[5] effect, Because of that effect the calculation of Key Points may not be accurate. So smoothing is done by Converting the image from Raster Scale to Real Plane.

\subsection{Harris Corner Point Detection}

Harris [6] is one of the widely used corner detection algorithms which is based on intensity and considered a gray scale image. A small window image patch is sweeped along the right direction [5] of the image. According to the change in intensity of the image, corner point is detected. If the window image patch is flat then all shifts will result in only a small change. If the windowed patch is a corner or isolated point, and then all shifts will result in large change. A corner can thus be detected by finding, when the minimum change produced by any of the shifts is large.-

\subsection{Problem Definition-}

The problem is categorized into two phases i.e., Phase I 
$\&$

Phase II

2.3.1. Phase I: The goal is to trace the contour to extract their Boundary pixels.

2.3.2. Phase II: The goal is to find the Interest Points by utilizing the boundary pixels.

\section{PROPOSED WORK}

\subsection{Proposed Algorithm for Interest Point Detection}

1. Begin Algorithm key point detection.

2. Considering the second point $\mathrm{p} 2$ as the start point.

3. Repeat step 3 to 4 for each point in the ordered set points until we reach the next point as the start point.

4. Find the angle between the two straight line formed by joining the adjacent points $\mathrm{p} 1$ and $\mathrm{p} 3$ with $\mathrm{p} 2$.
5. The deflection angle thus considered by taking the absolute value of the acute angle in an array. Find out the standard deviation of the angles and considered the result as the threshold value.

6. Considering the $\mathrm{p} 1$ point as the starting point.

7. Repeat step 8 to 10 for each point in the ordered set points until we reach the next point as the start point.

8. If the deflection angle of the point is greater than the threshold value then store the point as a key point.

9. If key point $=\mathrm{p} 2$ then set $\mathrm{p} 1$ to $\mathrm{p} 2, \mathrm{p} 2$ to $\mathrm{p} 3$ and p3 to the next point.

10. If $\mathrm{p} 2$ is not a key point then set $\mathrm{p} 2$ to $\mathrm{p} 3$ and $\mathrm{p} 3$ to the next point, keeping p1 unchanged.

11. End

4. RESULT ANALYSIS

4.1. Comparative Result

Table 1: Ground Truth VS Proposed Algorithm

\begin{tabular}{|c|c|c|c|}
\hline GROUND TRUTH & GROUND TRUTH & EXPERIMENT & EXPERIMENT \\
\hline $\mathrm{X} 1$ & Y1 & $\mathrm{X} 2$ & Y2 \\
\hline 50 & 80 & 50 & 82 \\
\hline 53 & 92 & 54 & 92 \\
\hline 73 & 113 & 72 & 117 \\
\hline 54 & 182 & 54 & 180 \\
\hline 51 & 200 & 49 & 201 \\
\hline 19 & 216 & 21 & 216 \\
\hline 20 & 250 & 20 & 250 \\
\hline 22 & 242 & 21 & 240 \\
\hline 7 & 276 & 8 & 272 \\
\hline 15 & 282 & 13 & 283 \\
\hline 24 & 290 & 25 & 290 \\
\hline 34 & 288 & 36 & 291 \\
\hline 43 & 292 & 39 & 292 \\
\hline 54 & 288 & 53 & 288 \\
\hline 44 & 275 & 44 & 272 \\
\hline
\end{tabular}




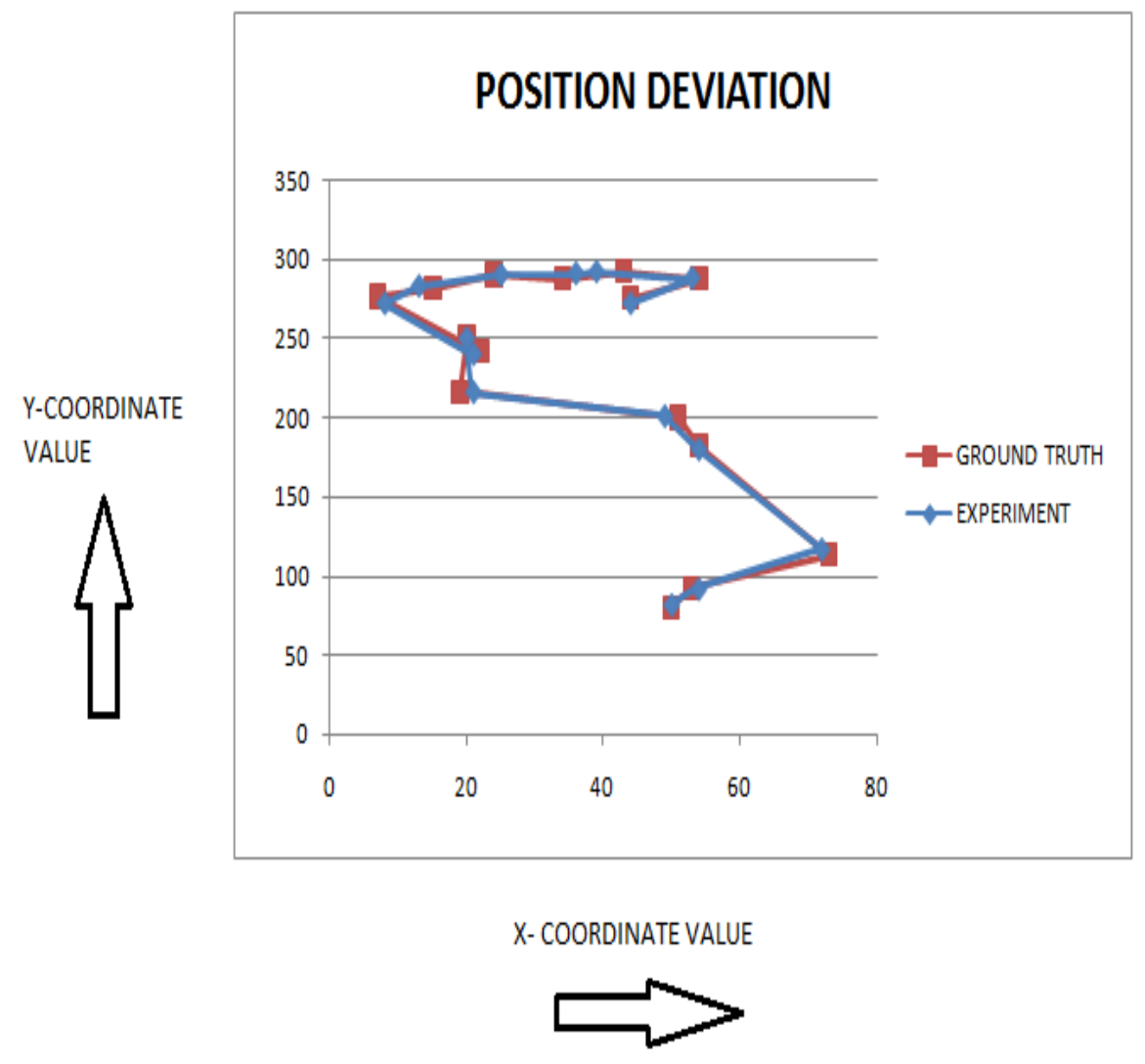

Figure 1: Key Point position deviation of Proposed Algorithm

\subsection{Analysis Of Parameter Values}

Table 2: NUMBER OF BOUNDARY POINTS - Data Sets

\begin{tabular}{|c|c|}
\hline NO OF BOUNDARY POINTS & PERFORMANCE IN PERCENTAGE \\
\hline 10 & 100 \\
\hline 20 & 99 \\
\hline 30 & 99 \\
\hline 40 & 98 \\
\hline 50 & 98 \\
\hline 70 & 97 \\
\hline 100 & 97 \\
\hline 150 & 94 \\
\hline 200 & 92 \\
\hline 250 & 90 \\
\hline 300 & 89 \\
\hline 350 & 89 \\
\hline 400 & 88 \\
\hline
\end{tabular}




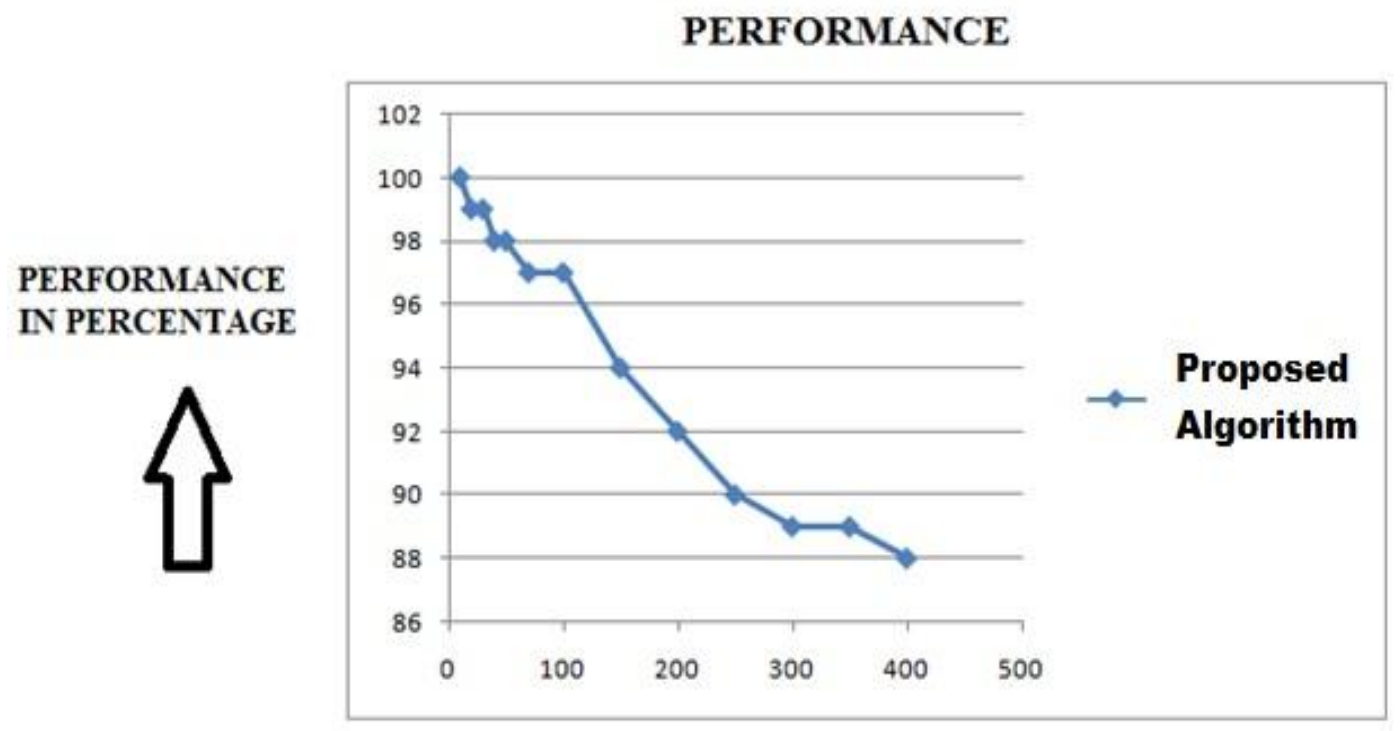

NUMBER OF BOUNDARY POINTS

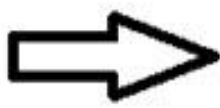

Figure 2: Performance in percentage: Proposed Algorithm

\section{CONCLUSION}

This work has been accomplished to find the key points [7] by which a contour of the image can be extracted. So, to find the interest points [7] first the contour is traced to get the ordered pair of pixels. The analysis reveals that the selection probability of this algorithm is good enough to determine the point as a key point. Although the selection probability decreases when the deflection angle increases, still it provides a better probability of selection. This technique has an advantage of good recognition accuracy rate and it's a lossless technique for matching [8].

\section{REFERENCES}

[1] Erik G. Learned-Miller, Introduction to Computer Vision, January 19, 2011.

[2] R.Owens,"Lecture 6",Computer Vision IT412,10/29/1997. http://homepages.inf.ed.ac.uk/rbf/CVonline/LOCAL _COPIES/OWENS/LECT6/node2.html Abeer George Ghuneim, Contour Tracing, 2000
[3] Fu Chang, Chun-Jen Chen and Chi-Jen Lu, A linear-time component-labeling algorithm using contour tracing technique, Received 8 August 2003; accepted 8 September 2003

[4] Young Hoon Lee ,Tung-Sing Leung, Gérard Medioni , Real-time staircase detection from a wearable stereo system.

[5] Konstantinos G.Derpanis, The Harris Corner Detector, October 27,2004.

[6] CORDELIA SCHMID, ROGER MOHR AND CHRISTIAN BAUCKHAGEINRIA Rh^one-Alpes, 655 av. de l'Europe, 38330 Montbonnot, France, Evaluation of Interest Point Detectors.

[7] Stefan Leutenegger, Image Keypoint Detection, Description, and Matching.

[8] M. Zukal and X. Qui, "Algorithms for interest points detection," in 6th International Conference on Teleinformatics 2011, 2011, pp. 163167,C.Schmid,R.Mohr and C.Bauckhage,"Evaluation of interest point detectors," Int. J. Comput. Vision, vol. 37, pp. 151172, June 2000. 